\title{
DNA damage and gene transcription: accident or necessity?
}

\author{
Cell Research (2015) 25:769-770. doi:10.1038/cr.2015.71; published online 9 June 2015
}

\begin{abstract}
The extent to which DNA repair machinery facilitates gene activation remains poorly appreciated. A new study published in Cell Research reports a novel function of $\mathrm{H} 2 \mathrm{AX}$, a substrate of ATM and known DNA damage marker, in transcriptional initiation.
\end{abstract}

In eukaryotic cells, genomic DNA is organized in chromatin. The repeating unit of chromatin is the nucleosome core particle containing histones with bound DNA. However, chromatin also contains non-histone chromatinassociated proteins; among them the high mobility group (HMG) proteins are the most abundant. HMGs can act both as activators and repressors of gene expression, and most likely facilitate DNA conformational changes by stabilizing specialized nucleoprotein structures at promoter and enhancer regions of regulated genes. Experimental evidence suggests that HMGs compete with linker histone $\mathrm{H} 1$ for the binding to the chromatin fiber [1]. As H1 is involved in the compaction of the chromatin and is believed to be a general repressor, its replacement by HMGs could alter chromatin domain structures. To date, however, the processes underlying transcription regulation by HMG proteins remain poorly understood.

Eukaryotic transcriptional initiation is a complex process requiring multiple enzymatic activities, and there have been reports suggesting a high frequency of DNA damage during transcriptional activation. However, a clear mechanistic link between these two processes has not yet been established. The Rosenfeld group reported that gene activation by nuclear receptors in MCF7 breast cancer cells requires topoisomerase IIb (Topo IIb) binding to the target promoters to generate a transient double-stranded DNA break (DSB). The resulting activation of PARP-1 and the subsequent recruitment of components of the DNA repair machinery by nuclear receptor co-activator $\mathrm{ASC} 2$ are required for a nucleosome-specific exchange of histone $\mathrm{H} 1$ by HMGB1/2 and chromatin relaxation [2]. More recently, the same group reported that translocations involving TMPRSS2, ERG, and ETV1 in prostate cancer require the androgen receptor (AR), which can promote sitespecific DSBs and favor chromosomal translocation. Thus, the authors propose that liganded AR acts as a mutagen, which recruits the enzymatic machineries activated by genotoxic stress [3]. Another postulated mechanism links DNA repair to transcription through the cleavage of DNA by hydrogen peroxide produced by the histone demethylase LSD1, which results in recruitment of 8-oxoguanine-DNA glycosylase 1 as well as Topo IIb and triggers chromatin and DNA conformational changes essential for estrogen-induced transcription [5]. The generality of this proposed mechanism remains to be confirmed.

In a recently published study in Cell Research, Singh and collaborators [6] provide further evidence supporting a connection between transcription and DNA repair in the context of the TGF $\beta 1$ signaling during epithelial-mesenchymal transition (EMT). The authors use a combination of proteomics-based interactome analysis, ChiP-seq and expression microarrays to propose a novel mechanism of transcriptional activation, which requires HMGA2 and the well-established DNA damage marker H2AX phosphorylated by ATM at $\mathrm{S} 139(\gamma-\mathrm{H} 2 \mathrm{AX})$ (Figure 1). Although the recruitment of kinases to chromatin after external stimuli has been previously shown [7], the role of ATM and $\mathrm{H} 2 \mathrm{AX}$ in transcriptional activation is novel and exciting. These results suggest that controlled chromatin dynamics during transcription might involve DNA breaks, which would in turn require tight regulation of repair mechanisms to ensure the integrity of the genome is maintained. This is very interesting proposal that opens the door for future research.

One possible future direction may be to explore the nature of the nucleosome changes associated with DSB, HMGA2 and of H2AX. Although it has been proposed that $\mathrm{H} 2 \mathrm{AX}$ and its phosphorylation in S139 alters nucleosome stability [8], other reports argue against a general role for the $\mathrm{C}$-terminal tail of H2AX in chromatin organization and suggest that $\gamma$-H2AX acts by recruitment of repair components rather than by destabilizing chromatin structures [9]. An attractive hypothesis is that ATM-dependent phosphorylation of $\mathrm{H} 2 \mathrm{AX}$ along with $\mathrm{H} 1$ phosphorylation, and/or its PARylation [4], could weaken the chromatin binding of $\mathrm{H} 1$ and thus favor HMGA2 loading and subsequent transcriptional activation (Figure 1). The requirement of PARP1 enzyme activity for gene regulation by nuclear receptors has been confirmed in genome wide studies, which also showed a direct activation of PARP1 via CDK2-mediated phosphorylation [4].

Together these results offer a possible mechanistic link between gene activa- 

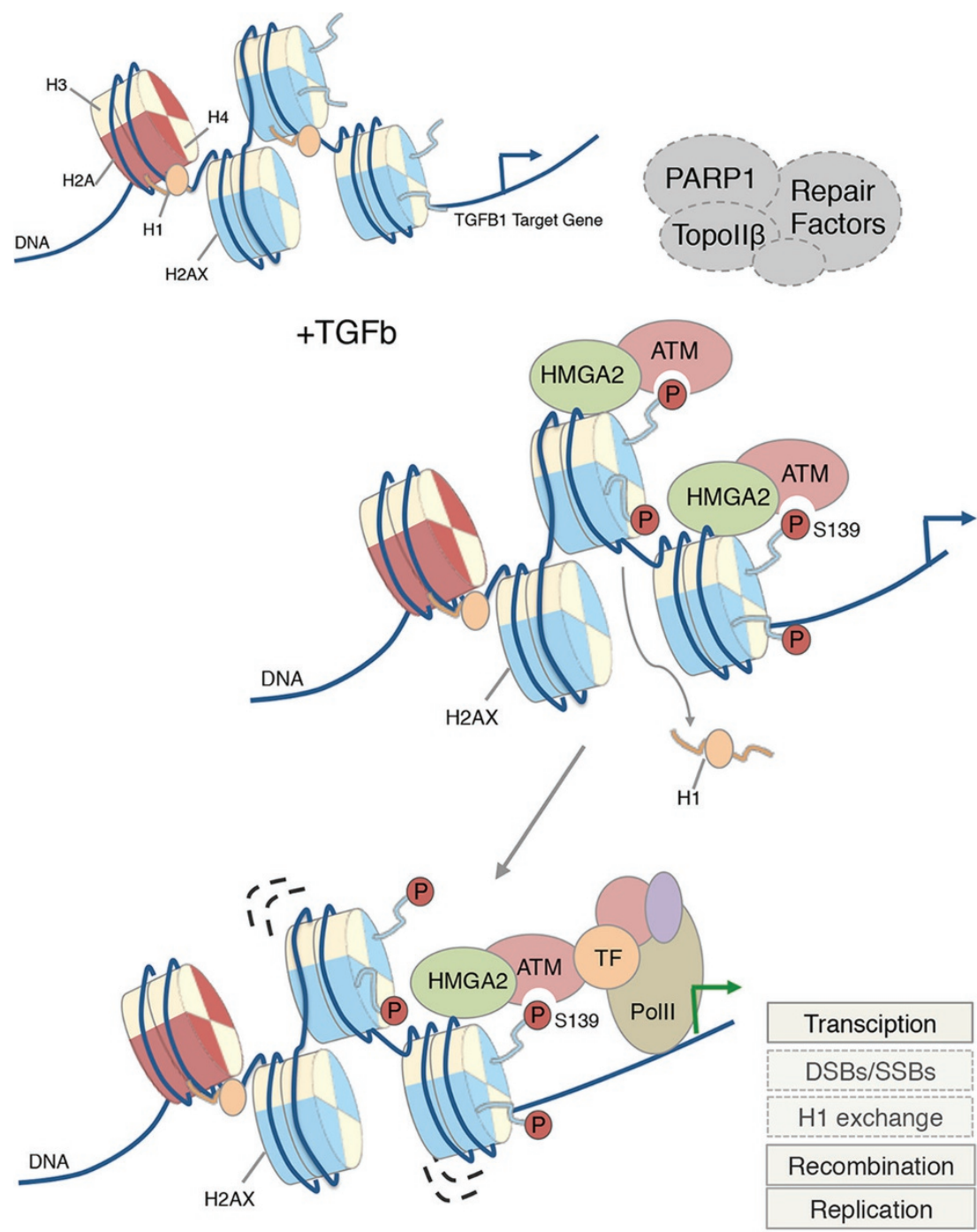

Figure 1 TGF $\beta 1$-induced transcription requires HMGA2 and ATM-mediated phosphorylation of $\mathrm{H} 2 \mathrm{AX}$. Binding of HMGA2 could promote $\mathrm{H} 1$ displacement and concomitantly change the structure of the chromatin at the target sites and thus facilitate gene activation. Other repair-associated enzymes as PARP1 and TOPOIlb, which hypothetically could be involved in this process, are also depicted. tion and components of the DNA damage/repair machinery. However, further experiments are needed to discriminate between the differential roles of Topo II, DSB, histone $\mathrm{H} 1, \mathrm{HMG}$ and the histone variant $\mathrm{H} 2 \mathrm{AX}$ in these two intermingled cellular processes. A more in-depth analysis will shine some light on still hidden aspects of mechanisms by which eukaryotic cells manage to control gene regulation, while maintaining the integrity of their genomic information.

\section{Miguel Beato ${ }^{1}$, Roni H Wright ${ }^{1}$, Guillermo P Vicent ${ }^{1}$}

${ }^{I}$ Center for Genomic Regulation (CRG), Dr Aiguader 88, E-08003, Barcelona, Spain; University Pompeu Fabra (UPF), E-08803, Barcelona, Spain Correspondence: Miguel Beato ${ }^{\mathrm{a}}$,

Guillermo P Vicent ${ }^{\mathrm{b}}$

aE-mail: miguel.beato@crg.es

bE-mail: guillermo.vicent@crg.es

\section{References}

1 Catez F, Yang H, Tracey KJ, et al. Mol Cell Biol 2004; 24:4321-4328.

2 Ju BG, Lunyak VV, Perissi V, et al. Science 2006; 312:1798-1802.

3 Lin C, Yang L, Tanasa B, et al. Cell 2009; 139:1069-1083.

4 Wright RH, Castellano G, Bonet J, et al. Genes Dev 2012; 26:1972-1983.

5 Perillo B, Ombra MN, Bertoni A, et al. Science 2008; 319:202-206.

6 Singh I, Ozturk N, Cordero J, et al. Cell Res 2015; 25:837-850.

7 Vicent GP, Ballare C, Nacht AS, et al. Mol Cell 2006; 24:367-381.

8 Li A, Yu Y, Lee SC, et al. J Biol Chem 2010; 285:17778-17788.

9 Fink M, Imholz D, Thoma F. Mol Cell Biol 2007; 27:3589-3600. 